\title{
V838 Monocerotis: the central star and its environment a decade after outburst
}

\author{
O. Chesneau ${ }^{1,+}$, F. Millour ${ }^{1}$, O. De Marco $^{2}$, S. N. Bright ${ }^{1,2}$, A. Spang ${ }^{1}$, D. P. K. Banerjee ${ }^{3}$, N. M. Ashok ${ }^{3}$ \\ T. Kamiński ${ }^{4}$, J. P. Wisniewski ${ }^{5}$, A. Meilland ${ }^{1}$, and E. Lagadec ${ }^{1, \star}$ \\ ${ }^{1}$ Laboratoire Lagrange, UMR 7293, Univ. Nice Sophia-Antipolis, CNRS, Observatoire de la Côte d'Azur, 06300 Nice, France \\ e-mail: Florentin.Millour@oca.eu \\ 2 Department of Physics \& Astronomy, Macquarie University, Sydney, NSW 2109, Australia \\ 3 Physical Research Laboratory, Navrangpura, Ahmedabad, 380009 Gujarat, India \\ ${ }^{4}$ Max-Planck Institut für Radioastronomie, Auf dem Hügel 69, 53121 Bonn, Germany \\ 5 HL Dodge Department of Physics \& Astronomy, University of Oklahoma, 440 W Brooks Street, Norman, OK 73019, USA
}

Received 24 June 2014 / Accepted 21 July 2014

\begin{abstract}
Aims. V838 Monocerotis erupted in 2002, brightened in a series of outbursts, and eventually developed a spectacular light echo. A very red star emerged a few months after the outburst. The whole event has been interpreted as the result of a merger.

Methods. We obtained near- and mid-IR interferometric observations of V838 Mon with the AMBER and MIDI recombiners located at the Very Large Telescope Interferometer (VLTI) array. The MIDI two-beam observations were obtained with the $8 \mathrm{~m}$ unit telescopes between October 2011 and February 2012. The AMBER three-beam observations were obtained with the compact array $(B \leq 35 \mathrm{~m})$ in April 2013 and the long array $(B \leq 140 \mathrm{~m})$ in May 2014, using the $1.8 \mathrm{~m}$ auxiliary telescopes.

Results. A significant new result is the detection of a compact structure around V838 Mon, as seen from MIDI data. The extension of the structure increases from a FWHM of 25 mas at $8 \mu \mathrm{m}$ to 70 mas at $13 \mu \mathrm{m}$. At the adopted distance of $D=6.1 \pm 0.6 \mathrm{kpc}$, the dust is distributed from about 150 to $400 \mathrm{AU}$ around V838 Mon. The MIDI visibilities reveal a flattened structure whose aspect ratio increases with wavelength. The major axis is roughly oriented around a position angle of $-10^{\circ}$, which aligns with previous polarimetric studies reported in the literature. This flattening can be interpreted as a relic of the 2002 eruption or as caused by the influence of the currently embedded B3V companion. The AMBER data provide a new diameter for the pseudo-photosphere, which shows that its diameter has decreased by about $40 \%$ in $10 \mathrm{yr}$, reaching a radius $R_{*}=750 \pm 200 R_{\odot}(3.5 \pm 1.0 \mathrm{AU})$.

Conclusions. After the 2002 eruption, which was interpreted as the merging of two stars, it seems that the resulting source is relaxing to a normal state. The nearby environment exhibits an equatorial overdensity of dust up to several hundred AU.
\end{abstract}

Key words. techniques: high angular resolution - stars: individual: V838 Monocerotis - circumstellar matter - stars: mass-loss

\section{Introduction}

V838 Mon is a nova-like object that erupted in 2002 in a series of outbursts (reaching $V \sim 6.8$ ). It subsequently developed a light echo that was extensively studied by Bond et al. (2003), Banerjee et al. (2006), and Tylenda \& Kamiński (2012). The eruption was unlike classical novae because the effective temperature of the object dropped and the spectral type evolved into a very late L-type supergiant (Evans et al. 2003; Loebman et al. 2014). The event has been interpreted as the merger of a star of $\sim 8 M_{\odot}$ with a subsolar mass star (Soker \& Tylenda 2003; Tylenda et al. 2005; Tylenda \& Soker 2006). The mid-IR flux of V838 Mon increased by a factor of two between 2004 and 2007, suggesting that new dust was forming in the expanding ejecta of the outbursts (Wisniewski et al. 2003a; Tylenda 2005; Wisniewski et al. 2008). The expanding ejecta engulfed a companion close to the central source (Bond 2006; Munari et al. 2007; Kolka et al. 2009).

* Based on observations at Paranal Observatory under programs 088.D-0005, 090.D-0011, 091.D-0030 and 093.D-0056.

${ }^{\dagger} \mathrm{O}$. Chesneau passed away shortly before submitting this Letter. We express our profound sadness on this premature demise and convey our deepest condolences to his family.
The progenitor of V838 Mon was found in a variety of surveys, including $2 \mathrm{MASS}$, and appears consistent with a somewhat reddened early main-sequence star. Post-outburst observations have found a faint, blue component in the spectrum, and Tylenda et al. (2005) argued that the pre-outburst spectral energy distribution (SED) is well-matched by a pair of early main-sequence stars $(\mathrm{B} 3 \mathrm{~V}+\mathrm{B} 1.5 \mathrm{~V}$ or B4V + A0.5V), making V838 Mon a triple system de facto (main star + subsolar merging star $+\mathrm{B} 3 \mathrm{~V}$ companion). Lynch et al. (2004) obtained numerous IR spectra of V838 Mon during and after the eruption (2002-2003). They fit their data to a model consisting of a cool $\left(T_{\mathrm{eff}}=2100 \mathrm{~K}\right.$, $R_{*}=8.8 \mathrm{AU}$ ) stellar photosphere surrounded by a large, absorbing molecular cloud. A more recent work (Loebman et al. 2014) provides precise estimates of the stellar and enshrouding cloud parameters, with an effective temperature of the star of 2000-2200 K, and a radius of the shell of $R=263 \pm 10 \mathrm{AU}$.

Lane et al. (2005) first used long-baseline near-IR $(2.2 \mu \mathrm{m}$ baselines lower than $85 \mathrm{~m}$ ) interferometry in NovemberDecember 2004 to provide the first direct measurement of the angular size of V838 Mon using the two-telescope recombiner Palomar Testbed Instrument (PTI). At this epoch, they measured an angular diameter for the central source of $\Theta=1.83 \pm$ 0.06 mas. Reasonably accurate distances were determined from 
the light echo and using other methods (Munari et al. 2005; Sparks et al. 2008; Kamiński et al. 2011). We adopt the distance $D=6.1 \pm 0.6 \mathrm{kpc}$ from Sparks et al. (2008). Using this distance, the interferometric diameter translates into a linear radius for the supergiant of $1200 \pm 150 R_{\odot}(5.6 \pm 0.7 \mathrm{AU})$. Lane et al. (2005) were also to fit the data assuming an elliptical structure with a major axis oriented at PA $15^{\circ}$ whose extensions are $3.57 \times 0.07$ mas (i.e., the minor axis is unresolved).

V838 Mon is embedded in a dense, large-scale environment (Kamiński et al. 2011; Tylenda \& Kamiński 2012; Exter et al. 2014) that potentially contaminates observations of the central star. High angular resolution studies with a field of view (FOV) of a few arcsec are an asset in that domain to isolate the central regions from the extended dusty cloud. A great advantage of optical interferometry studies is to fully isolate the measurements from the extended environment.

This letter presents optical interferometry measurements obtained with the Very Large Telescope Interferometer (VLTI). The observations are presented in Sect. 2. In Sect. 3 we analyze the MIDI mid-IR measurements followed by the AMBER $H$ - and $K$-band measurements by means of simple geometrical models. The results are then discussed in Sect. 4.

\section{Observations}

Mid-IR interferometric data were obtained with the twotelescope recombiner MIDI (Leinert et al. 2004) between October 2011 and February 2012 using the $8 \mathrm{~m}$ unit telescopes (UTs) of the Very Large Telescope. These observations provided dispersed fringe visibilities between 8 and $13 \mu \mathrm{m}$ in addition to classical spectrophotometric capabilities.

Near-IR observations were obtained with AMBER (a threetelescope combiner located at the VLTI: Petrov et al. 2007) in November 2012 and February 2013, using the low spectral resolution mode $(R=35)$ with the UTs. Unfortunately, these data are unusable because of an unexpectedly resolved calibrator (see details in the Appendix) and are therefore not presented here. In April 2013, observations were repeated with the $1.8 \mathrm{~m}$ auxiliary telescopes (ATs) under photometric conditions, using the compact configuration $(B \leq 35 \mathrm{~m})$ and more suitable calibrators. The extended configuration $(B \leq 140 \mathrm{~m})$ was subsequently used in May 2014 to observe V838 Mon.

The great advantage of both instruments is that the FOV of the interferometric measurements are within the point spread function of a single telescope, that is, the same value of $\sim 0.3^{\prime \prime}$ at $2.2 \mu \mathrm{m}$ for the $1.8 \mathrm{~m}$ ATs with AMBER, and at $10 \mu \mathrm{m}$ for the $8 \mathrm{~m}$ UTs with MIDI. This isolates the central source from the extended emission of the cloud in which V838 Mon is embedded. The observation log is presented in Table 1 and the $(u, v)$ plane coverage is plotted in Fig. 1.

We reduced the AMBER data using the standard datareduction software amdlib v3.0.8 (Tatulli et al. 2007; Chelli et al. 2009). Near-IR $J H K$ photometry was obtained on a regular basis from the $1.2 \mathrm{~m}$ telescope at the Mt. Abu Observatory, India (Banerjee \& Ashok 2012). These measurements helped to prepare the interferometric observations. On March 1, 2013, the $J, H, K$ band magnitudes were $7.12,5.91,5.43$, respectively, almost unchanged since November 2011.

Visibilities are a proxy to the object's size and shape. The AMBER visibilities are presented in Fig. 2 at selected wavelengths for all baselines, and for the longest baseline in Fig. 3 together with the $J H K$ Mt. Abu spectrum. Strong variations as a function of wavelength are seen, especially at the edge of the
Table 1. Log of AMBER and MIDI observations of V838 Mon.

\begin{tabular}{lcccc}
\hline \hline Date & Stations & Calibrators & Wavelength & Nb. obs. \\
\hline MIDI & & & & \\
$10 / 10 / 2011$ & U3-U4 & $1^{\circ}$ & $8-13 \mu \mathrm{m}$ & 2 \\
$13 / 12 / 2011$ & U2-U3 & $1^{\circ}$ & $8-13 \mu \mathrm{m}$ & 1 \\
$14 / 12 / 2011$ & U2-U3 & $1^{\circ}$ & $8-13 \mu \mathrm{m}$ & 1 \\
$11 / 02 / 2012$ & U3-U4 & $1^{\circ}$ & $8-13 \mu \mathrm{m}$ & 1 \\
AMBER & & & & \\
$14 / 04 / 2013$ & A1-B2-D0 & $2^{\triangleleft}, 3^{\triangleright}$ & $1.54-2.50 \mu \mathrm{m}$ & 3 \\
$15 / 04 / 2013$ & A1-C1-D0 & $2^{\triangleleft}, 4^{\triangleleft}$ & $1.56-2.50 \mu \mathrm{m}$ & 2 \\
$06 / 05 / 2014$ & A1-G1-J3 & $5^{\triangleright}$ & $1.53-2.46 \mu \mathrm{m}$ & 1 \\
\hline
\end{tabular}

Notes. Calibrator angular diameters from SearchCal/JMMC (Bonneau et al. 2006) and getCal/NExScI. ( ${ }^{(\circ)} \mathrm{HD} 526662.53 \pm 0.21$ mas;

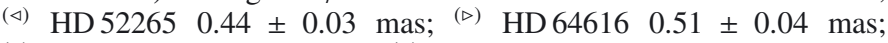
${ }^{(\triangle)}$ HD $636600.96 \pm 0.07$ mas; ${ }^{(\nabla)}$ HD $549900.57 \pm 0.59$ mas.
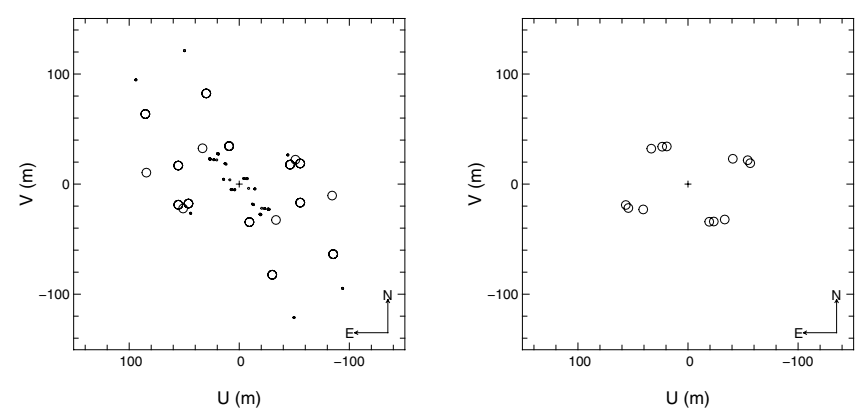

Fig. 1. Left: $(u, v)$ coverage of the AMBER observations. Small dots are observations with ATs and larger circles with UTs. Right: $(u, v)$ coverage of the MIDI observations.

$H$ and $K$ bands, where they decrease relative to the band centers $(1.7 \mu \mathrm{m}$ and $2.2 \mu \mathrm{m})$. All baseline lengths show this curved shape. We are confident that this is not a flux bias or instrumental effect, but comes from the source itself.

Closure phases can be qualitatively interpreted as a proxy to asymmetries: a nonzero (modulo $\pi$ ) closure phase is a sign of asymmetries in the object's shape. The 2014 closure phases are of good quality and compatible with zero with a mean value of $-1.04 \pm 1$. 12 . The object is therefore likely centro-symmetric as seen by AMBER. The spectral range of the AMBER data is $1.5-2.5 \mu \mathrm{m}$.

The MIDI data were processed using the software mia+ews ${ }^{1}$ (Chesneau 2007). The data were secured in High_Sens mode, implying that the photometry is obtained in a sequence, subsequently to fringes. The quality of the data ranges from high quality (error level $\sim 10 \%$ ) to poor quality for some baselines (error level reaching $\sim 25 \%$ ). The spectrophotometric calibration of the mid-IR flux was performed with the calibrator HD 52666 (M2III, IRAS F12 = $18.3 \mathrm{Jy}$ with a $5 \%$ error). The ten MIDI spectra from two telescopes were merged, which led to a spectrophotometry with an $8 \%$ error level. This represents the midIR flux of the source between October and February 2011. The spectrum was compared with measurements at the same epoch from the $\mathrm{WISE}^{2}$ and $\mathrm{AKARI}^{3}$ satellites obtained with much larger apertures. The flux in the WISE filter $(\lambda=11.56 \mu \mathrm{m}$, $\Delta \lambda=5.51 \mu \mathrm{m})$ is $31.56 \pm 0.06 \mathrm{Jy}$ and in the AKARI filter $(\lambda=8.22 \mu \mathrm{m}, \Delta \lambda=4.10 \mu \mathrm{m})$ the flux is $20.11 \pm 0.39 \mathrm{Jy}$. The

\footnotetext{
http://home.strw. leidenuniv.nl/ nevec/MIDI/

2 Taken on April 2 and October 10, 2010.

3 Obtained between May 2006 and August 2007.
} 
O. Chesneau et al.: V838 Mon 10 years after outburst

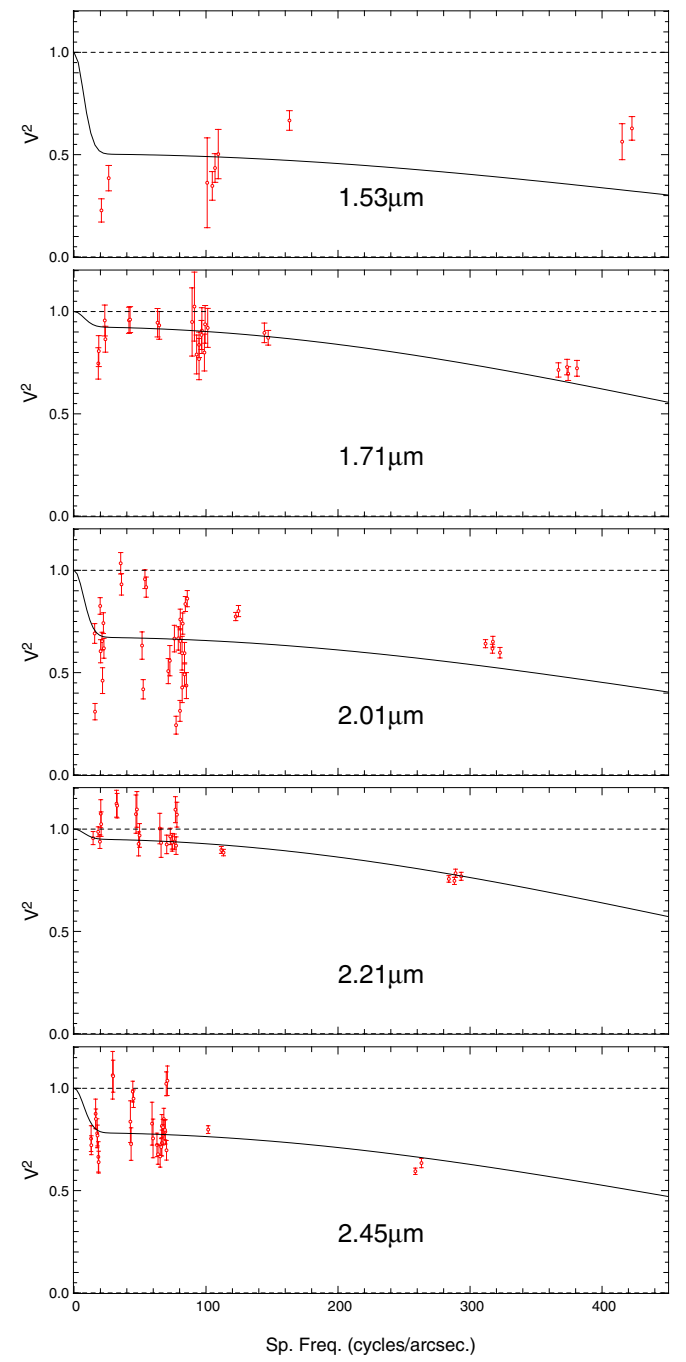

Fig. 2. AMBER data shown as a function of spatial frequencies for selected wavelengths. The black line shows our best-fit model of a uniform disk plus an extended component (50 mas FWHM here).

spectrophotometry and the corresponding Gaussian estimates from the MIDI are shown in Fig. 4.

\section{Analysis}

The MIDI dispersed visibilities were translated into the simplest possible geometrical adhoc model - a Gaussian brightness distribution, as described in Leinert et al. (2004). The resulting fits shown in Fig. 4 depict the general appearance of the mid-IR structure. The semimajor and semiminor axes of the drawn ellipses represent the half-width at half maximum (HWHM) of the Gaussian. The extent of that structure increases from a FWHM of 25 mas at $8 \mu \mathrm{m}$ to 70 mas at $13 \mu \mathrm{m}$, with a high flattening ratio. Despite the heterogeneity of the quality of the MIDI visibilities, the flattening of that structure is certain, but we can only provide a loose range for flattening ratio values between 1.1 and 2.5 near $9 \mu \mathrm{m}$, and between 1.3 and 60 near $12 \mu \mathrm{m}$. The major axis is oriented at a PA close to $-10^{\circ}$ with an uncertainty of more than $\pm 30^{\circ}$. The flattening increases with the wavelength, from a roughly round structure at $8 \mu \mathrm{m}$ to a very flattened structure at $13 \mu \mathrm{m}$. This may come from the contribution of the star, which is stronger at $8 \mu \mathrm{m}$. The flux from WISE agrees fairly well with the MIDI flux. AKARI data are offset in time from the MIDI data by five to six years. Given that the source's SED has been
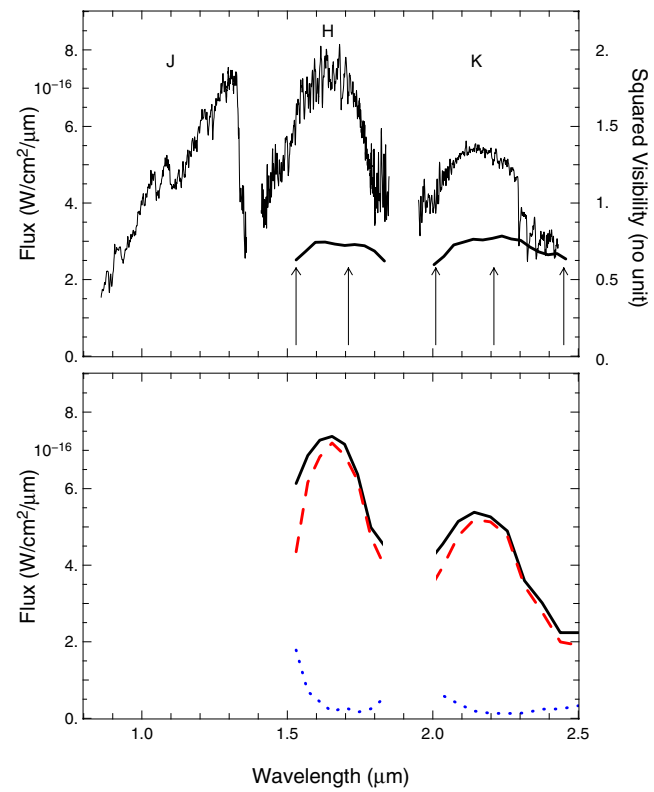

Fig. 3. Top: $0.85-2.5$ micron spectrum of V838 Mon on 11 December 2013 corrected for extinction using $E(B-V)=0.5$. For comparison, we show the AMBER longest baseline visibility (thick black lines, right axis), and indicate with arrows the wavelengths of Fig. 2. Bottom: spectra extracted from the AMBER data according to a downgraded resolution version of the above spectrum (black line). Red dashed line: the star spectrum. Blue dotted line: extended emission spectrum.

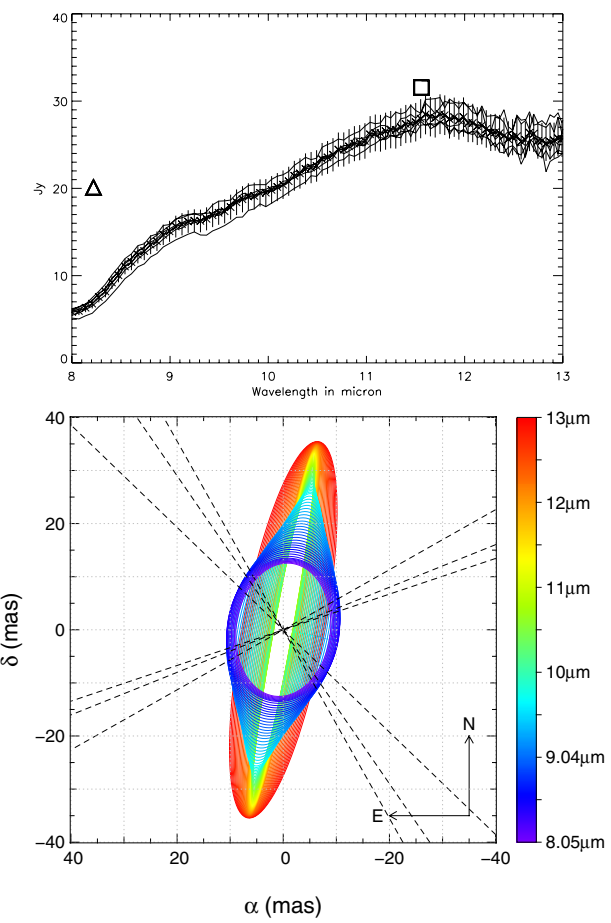

Fig. 4. Top: MIDI spectrum obtained in 2011. For comparison, we added AKARI (triangle) and WISE (square) fluxes. Bottom: 2D Gaussian fit to the whole MIDI dataset. The dashed lines represent the direction of the observation baselines.

evolving in the IR (Wisniewski et al. 2008) some difference in the value of the 9 micron flux measured by AKARI and MIDI is expected. This implies that most of the mid-IR flux in 2011 originates from the vicinity of the central star $\left(\leq 0.4^{\prime \prime}\right)$.

The 2013 short-baseline AMBER visibilities are all very close to unity in the band centers at $1.7 \mu \mathrm{m}$ and $2.2 \mu \mathrm{m}$ and are consistent with a completely unresolved object. The 
long-baseline, 2014 visibilities are close to $V^{2}=0.8$ at the same wavelengths.

The visibilities as a function of wavelength of the $H$ and $K$ bands decrease relative to the band centers $(1.7 \mu \mathrm{m}$ and $2.2 \mu \mathrm{m}$ ), as shown in Fig. 3. This is indicative of an object whose shape changes as a function of wavelength. At the same time, the visibilities at the edge of the bands $(1.5 \mu \mathrm{m}, 2.0 \mu \mathrm{m}$ and $2.4 \mu \mathrm{m}$ ) show a plateau-like shape as a function of spatial frequencies (see Fig. 2), which is indicative of an additional resolved component. We therefore analyzed first the $K$-band center using a single-component model, and then added an additional component to analyze the full AMBER dataset.

We used the software fitOmatic (Millour et al. 2009) to derive an angular diameter assuming a uniform-disk model around $2.2 \mu \mathrm{m}$. If we include all the 2013 (short baselines) and 2014 (long baselines) observations, we find a diameter of $1.17 \pm 0.37$ mas. If we keep only the 2014 data, we obtain a diameter of $1.15 \pm 0.20$ mas. We adopted the latter diameter of $1.15 \pm 0.20$ mas because the 2013 data (short baselines) do not resolve the central star. This measured diameter is significantly smaller than that published by Lane et al. (2005).

The AMBER closure phases are all equal to zero within the error bars. We can therefore constrain the flux of an hypothetical companion star in addition to the central merger. To achieve this, we added a point-source to our model and used a simulated annealing algorithm to constrain its parameters using the observed visibilities and closure phases and starting with random initial parameters. Our conclusion is that its flux was always lower than $3 \%$ of the total flux, meaning that there would be a difference of at least 4 mag between the primary and the companion. The AMBER observations do not exclude the presence of the companion, but place important constraints on its NIR contribution to the SED.

The decreasing visibilities at the edges of the $H$ and $K$ bands indicate the presence of extended emission with a varying spectrum (as shown in the lower panel in Fig. 3). We cannot constrain the exact size of this contribution because of the plateau-like shape of the visibilities as a function of spatial frequencies, but we can provide a lower limit of its size of $\theta \geq 20$ mas. We tentatively associate this emission with the MIDI elongated dusty structure. The extended component would correspond to the $H$ and $K$-bands contribution of the MIDI elongated structure. It is noteworthy that adding this additional source of emission does not significantly change the estimated diameter for the central star.

To complement these measurements, we used a $J H K$ spectrum of the star from Mt Abu (India), obtained on 11 December 2013, to derive a low-resolution spectrum of the central star and the extended emission (Fig. 3, lower panel). The stellar spectrum is typical of a cool luminous star (see Lançon \& Wood 2000; Banerjee \& Ashok 2002, for a comparison) with prominent firstovertone $\mathrm{CO}$ bands at $2.29 \mu \mathrm{m}$ and beyond. Similar, albeit weak, $\mathrm{CO}$ second overtone features are also seen in the $H$ band. The deep down-turns at the band edges are due to the presence of water in its atmosphere (Banerjee et al. 2005). Other molecular features of $\mathrm{VO}$ and $\mathrm{TiO}$ are also seen, including some rather uncommon bands of AlO. The spectrum of the extended structure shows emission bands at the $H$ and $K$ band edges, where the water absorption occurs in the total spectrum.

\section{Discussion}

The PA of the major axis, as inferred from the dusty flattened structure discovered by MIDI, is $\approx-10^{\circ}$. The MIDI size increase is expected for a non-truncated dusty disk. At the adopted distance, the dust is distributed between 150 and $400 \mathrm{AU}$ from V838 Mon. This also in agrees with the earliest accurate spectropolarimetric measurements reported by Wisniewski et al. (2003a,b). They reported an intrinsic polarization with a PA of $127^{\circ} .0 \pm 0.5$ interpreted as scattering by a disk with a major axis at a PA of $37^{\circ}$.

The 2004 PTI interferometric data had been interpreted as either a uniform disk or an elliptical model, both consistently explaining the data well. We note that the $\mathrm{PA}=15_{-27}^{+3}{ }^{\circ}$ derived from PTI is roughly aligned with our longest AMBER baselines, that is, we would be sensitive to the major axis size if the correct Lane et al. (2005) model had been that of an elongated structure. Our AMBER data suggest a different picture. The supergiant has decreased in angular size by $\approx 40 \%$ in $\approx 10$ years since the measurements of Lane et al. (2005). The linear radius is now estimated to $750 \pm 200 R_{\odot}(3.5 \pm 1.0 \mathrm{AU})$. This radius is similar to the famous M2 Iab supergiant Betelgeuse (Haubois et al. 2009, $\left.885 \pm 90 R_{\odot}\right)$. Such an evolution has already been suggested by (Geballe et al. 2007). This diameter decrease means the star's photosphere has shrunk during the intervening period with an approximate rate of $1 \mathrm{~km} \mathrm{~s}^{-1}$.

The good quality of the closure phases provides crucial constraints and excludes any secondary star brighter than a few percent of the total flux in the 6-600 AU separation range. But we caution that a $\mathrm{B} 3 \mathrm{~V}$ star whose $K$-band magnitude is fainter than 15 would be totally undetectable by the interferometer.

The AMBER interferometric observations obtained $10 \mathrm{yr}$ after PTI suggest a contraction of the central star by about $40 \%$, which is in line with the spectral change of V838 Mon toward a "normal" M-type supergiant. No clear sign of deformation of the photosphere is detected.

The extended emission found in the AMBER data is enhanced relative to the central star around the $\mathrm{CO}$ overtone absorption features and the $\mathrm{H}_{2} \mathrm{O}$ absorption at the band edges. This means that there is water emission around the star at an angular scale larger than 20 mas, and smaller than 250 mas (field of view of one telescope at $2.2 \mu \mathrm{m}$ ). We think this emission $H$ and $K$-band emission is linked with the MIDI elongated dusty structure.

Several hypotheses can be proposed to explain the detection of the flattened structure at large distance, as observed by MIDI and supported by AMBER.

The most likely hypothesis is that the flattened structure is simply the relic of the large dust formation event as a consequence of the merging of the two stars (Wisniewski et al. 2008, 2003a; Nicholls et al. 2013). The ejecta velocity was low at outburst time - lower than $200 \mathrm{~km} \mathrm{~s}^{-1}$ as derived from P-Cygni profiles by Lynch et al. (2004) from several lines. In the 10 years since outburst, ejected material would not have traveled beyond $400 \mathrm{AU}$. This scenario is compatible with our finding.

The interferometric observations may also be discussed in the context of the B3V companion discovered around V838 Mon (Desidera \& Munari 2002; Wagner \& Starrfield 2002; Munari et al. 2002, 2005) and observed in the post-outburst spectra. The presence of the companion would explain the X-ray activity discovered in the vicinity of V838 Mon (Antonini et al. 2010). It is important to note that the distance of this companion from the central star was investigated by Munari et al. (2007), and they estimated the smallest separation to about $28 \mathrm{AU}$. On the other hand, Tylenda et al. (2009) derived a separation of $\approx 250 \mathrm{AU}$. This is well within the separation range detectable by AMBER. The MIDI measurements suggest a large separation of about $100 \mathrm{AU}$. In 2009, the B3V hot signature was no longer 
observed (Kolka et al. 2009). We therefore propose that the companion is building a circumbinary disk via the Lagrangian point L2, as proposed in some observational and theoretical studies on other sources (Plets et al. 1995; Meilland et al. 2010; Millour et al. 2011).

To conclude, we find a flattened dusty structure around V838 Mon. We also find that the central star decreased its diameter by nearly $40 \%$ in 10 years.

The interferometric picture of V838 Mon as of today is the following: it is now slowly becoming an anonymous red supergiant, surrounded by a flattened, probably transitory, dusty environment extending up to several hundreds of AU.

Acknowledgements. O. Chesneau had wished to express his deepest gratitude to the hospital staff for their professionalism and devotion during the writing of this Letter. This Letter makes use of the CDS, the JMMC and NExScI services.

\section{Appendix A: Bad 2012 calibrator: a newly discovered binary star}

The selected calibrator for the 2012 observations, HD 45299, is a previously-unseen visual binary star (see Fig. A.1) that we detect at 16.0 mas separation, position angle (PA) $-67^{\circ}$ and $0.6 / 0.4$ relative fluxes.

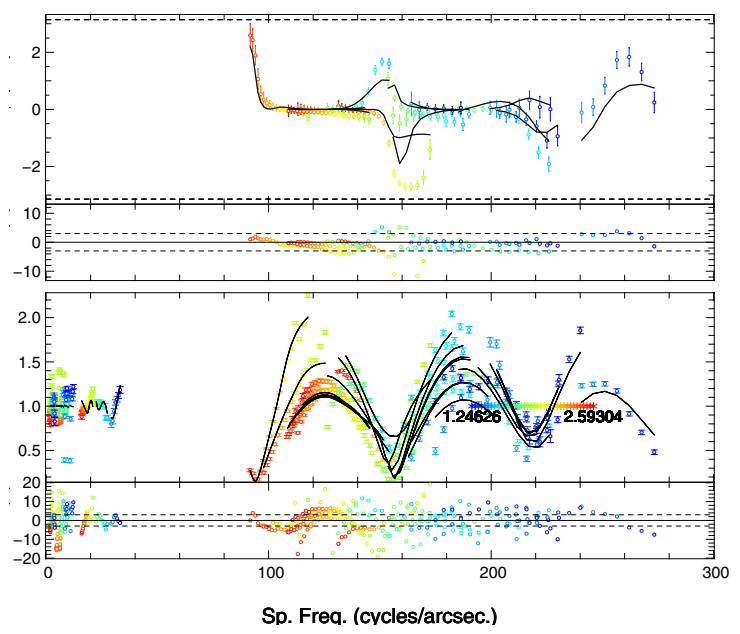

Fig. A.1. AMBER closure phases and differential visibilities (no calibrated $V^{2}$ available) for HD 45299 (color points with error bars) together with a best-fit model of a binary star (black lines). The spatial frequencies are projected along the binary direction $\left(-67^{\circ}\right)$ to show the binary modulation.

\section{References}

Antonini, F., Montez, Jr., R., Kastner, J. H., et al. 2010, ApJ, 717, 795

Banerjee, D. P. K., \& Ashok, N. M. 2002, A\&A, 395, 161

Banerjee, D. P. K., \& Ashok, N. M. 2012, Bull. Astron. Soc. India, 40, 243

Banerjee, D. P. K., Barber, R. J., Ashok, N. M., \& Tennyson, J. 2005, ApJ, 627, L141

Banerjee, D. P. K., Su, K. Y. L., Misselt, K. A., \& Ashok, N. M. 2006, ApJ, 644, L57

Bond, H. E. 2006, ATel, 966, 1

Bond, H. E., Henden, A., Levay, Z. G., et al. 2003, Nature, 422, 405

Bonneau, D., Clausse, J.-M., Delfosse, X., et al. 2006, A\&A, 456, 789

Chelli, A., Utrera, O. H., \& Duvert, G. 2009, A\&A, 502, 705

Chesneau, O. 2007, New Astron. Rev., 51, 666

Desidera, S., \& Munari, U. 2002, IAU Circ., 7982, 1

Evans, A., Geballe, T. R., Rushton, M. T., et al. 2003, MNRAS, 343, 1054

Exter, K., Cox, N. L. J., Decin, L., \& De Beck, E. 2014, in Asymmetrical Planetary Nebulae VI conference, Proc. conference held 4-8 November, 2013, eds. C. Morisset, G. Delgado-Inglada, \& S. Torres-Peimbert Geballe, T. R., Rushton, M. T., Eyres, S. P. S., et al. 2007, A\&A, 467, 269

Haubois, X., Perrin, G., Lacour, S., et al. 2009, A\&A, 508, 923

Kamiński, T., Tylenda, R., \& Deguchi, S. 2011, A\&A, 529, A48

Kolka, I., Liimets, T., Kankare, E., Pursimo, T., \& Datson, J. 2009, ATel, 2211, 1

Lançon, A., \& Wood, P. R. 2000, VizieR Online Data Catalog, J/A+AS/146/217 Lane, B. F., Retter, A., Thompson, R. R., \& Eisner, J. A. 2005, ApJ, 622, L137

Leinert, C., van Boekel, R., Waters, L. B. F. M., et al. 2004, A\&A, 423, 537

Loebman, S. R., Wisniewski, S. J., Schmidt, A. F., et al. 2014, ApJ, submitted Lynch, D. K., Rudy, R. J., Russell, R. W., et al. 2004, ApJ, 607, 460

Meilland, A., Kanaan, S., Borges Fernandes, M., et al. 2010, A\&A, 512, A73

Millour, F., Chesneau, O., Borges Fernandes, M., et al. 2009, A\&A, 507, 317

Millour, F., Meilland, A., Chesneau, O., et al. 2011, A\&A, 526, A107

Munari, U., Desidera, S., \& Henden, A. 2002, IAU Circ., 8005, 2

Munari, U., Henden, A., Vallenari, A., et al. 2005, A\&A, 434, 1107

Munari, U., Corradi, R. L. M., Henden, A., et al. 2007, A\&A, 474, 585

Nicholls, C. P., Melis, C., Soszyński, I., et al. 2013, MNRAS, 431, L33

Petrov, R. G., Malbet, F., Weigelt, G., et al. 2007, A\&A, 464, 1

Plets, H., Waelkens, C., \& Trams, N. R. 1995, A\&A, 293, 363

Soker, N., \& Tylenda, R. 2003, ApJ, 582, L105

Sparks, W. B., Bond, H. E., Cracraft, M., et al. 2008, AJ, 135, 605

Tatulli, E., Millour, F., Chelli, A., et al. 2007, A\&A, 464, 29

Tylenda, R. 2005, A\&A, 436, 1009

Tylenda, R., \& Kamiński, T. 2012, A\&A, 548, A23

Tylenda, R., \& Soker, N. 2006, A\&A, 451, 223

Tylenda, R., Soker, N., \& Szczerba, R. 2005, A\&A, 441, 1099

Tylenda, R., Kamiński, T., \& Schmidt, M. 2009, A\&A, 503, 899

Wagner, R. M., \& Starrfield, S. G. 2002, IAU Circ., 7992, 2

Wisniewski, J. P., Bjorkman, K. S., \& Magalhães, A. M. 2003a, ApJ, 598, L43

Wisniewski, J. P., Morrison, N. D., Bjorkman, K. S., et al. 2003b, ApJ, 588, 486

Wisniewski, J. P., Clampin, M., Bjorkman, K. S., \& Barry, R. K. 2008, ApJ, 683, L171 